\title{
CROSSING-OVER IN MICE HETEROZYGOUS FOR $t$-ALLELES
}

\author{
MARY F. LYON and RITA J. S. PHILLIPS \\ Medical Research Council Radiobiological Research Unit, \\ Harwell, Didcot, Berks
}

Received 2 I.iii. $5^{8}$

\section{INTRODUCTION}

For studies of mutagenesis in the house mouse it would be very useful if there were chromosome changes acting as crossover suppressors, which could be used to build up stocks resembling in function the GlB and Muller-5 stocks of Drosophila melanogaster. The only crossover suppressors so far known are some of the recessive lethal $t$-alleles in linkage group IX. The experiments reported in this paper were

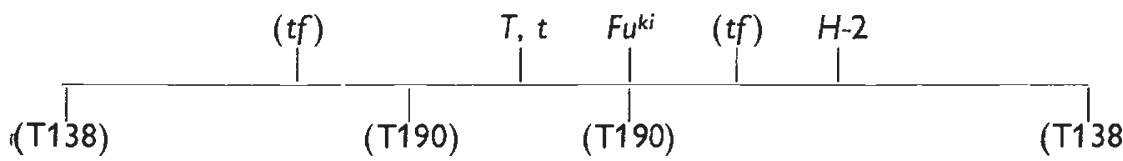

Fig.-Known loci in linkage group IX of the mouse.

$T$ Brachyury. Dominant. Heterozygote has short tail, homozygote dies in utero.

$t^{0}, t^{1}, \ldots, t^{n}$ Recessive alleles at the T-locus. $T t$ heterozygotes tailless, $+t$ normal. Homozygotes for some alleles lethal, some viable and normal tailed. Some alleles show non-Mendelian segregation in males, some suppress crossing-over.

$F u^{k i} \quad$ Kinky. Dominant. Heterozygote has kinky tail, homozygote dies.

$H$-2 Histocompatibility. Determines the antigenic specificity of tissues (e.g. tumours) as detected by transplantation studies.

tf Tufted. Recessive. Affects hair growth.

Tr38 Chromosome translocation involving linkage groups II and IX. The break in group IX is remote from 7 .

Trgo Chromosome translocation involving linkage groups IX and XIII. The break in group IX is near to $T$ and the translocated chromosome carries $t^{6}$.

The position of $t f, \mathrm{~T}_{\mathrm{r}_{3}} 8$ and $\mathrm{T}_{\mathrm{rg}} \mathrm{o}$ relative to $F u$ and $H_{-2}$ had not been found at the start of this work.

intended to extend knowledge of the crossover-suppressing effect of $t$-alleles in the hope of using them in stocks for studies of mutagenesis. The fig. gives a diagram of the loci in group IX as known at the start of these experiments and describes the genes and translocations concerned.

Dunn and Caspari (1945) and Dunn and Gluecksohn-Waelsch $(\mathrm{I} 953 b)$ showed that some $t$-alleles suppress crossing-over between $\mathcal{T}$ and the nearby locus $F u$. Until recently $\mathcal{T}, F u$ and $H-2$ were the only known loci in linkage group IX. This made more detailed studies of the crossover-suppressing effect of $t$-alleles technically difficult since $\mathcal{T}$ and $F u$ both affect the same organ, the tail, and the segregation of $H-2$ alleles can only be observed by a laborious technique of tumour transplantation. Recently, however, tufted, $t f$, was found to lie in this linkage group (Lyon, 1956). In addition translocation 
Tr38, described by Carter, Lyon and Phillips (1955, 1956), involves linkage groups II and IX, so that in mice carrying this translocation $\tau$ shows linkage with dilute, $d$, of group II.

The $t$-alleles used in the present experiments were $t^{6}$ and $t^{13}$. $t^{6}$ was found by Carter and Phillips (1950) and reported by Dunn and Gluecksohn-Schoenheimer (I950) not to differ from the previously known lethal allele, $t^{0}$, which is a crossover suppressor. The allele $t^{13}$ was found by. Dunn and Gluecksohn-Waelsch (1953a) in a normaltailed exception in a balanced lethal line $T t^{1} \times T t t^{1}$. It is viable when homozygous and its effect on crossing-over had not been tested at the start of this work. Crossing-over has been studied between the $T$-locus and $t f$ in $t^{6}$ and $t^{13}$ heterozygotes and among $T, t^{6}, t f$ and $d$ in $\mathrm{Tr}_{3} 8$ heterozygotes. In addition, a note is made of a possible simultaneous mutation and crossover in translocation Trgo which has a bearing on the observed high mutation rate of $t$-alleles (Dunn, i956).

\section{CROSSES AND RESULTS}

(i) Crossing-over in heterozygotes for $\mathrm{t} f$ and $\mathrm{t}^{6}$

A $T+t f t f$ female was crossed with a $+t^{6}++^{t f}$ male and $T t^{6}$ $+t f$ progeny were backcrossed to $++^{T}$ tftf animals. Offspring from these matings were used to make crosses of other types. The data are shown in table I. From $T t^{6}+t f$ heterozygotes there were no crossovers, while from $+t^{6}+t f$ heterozygotes two crossovers were obtained, one from a female and one from a male heterozygote. In this cross the presence or absence of the $t$-allele can only be scored in those young which inherited $T$ from their other parent. Hence, only young carrying $T$ give linkage information and the recombination in $+t^{6}+t f$ heterozygotes is $2 / 162$ or $\mathrm{I} \cdot 23$ per cent. with upper and lower fiducial limits at the 5 per cent. significance level of $0 \cdot I_{5}$ and $4 \cdot 46$ per cent. The data from males and females have been combined as no significant sex difference in recombination is indicated. Combining the data from male and female $+t^{6} / t f T$ heterozygotes, the upper fiducial limit of recombination at the 5 per cent. significance level is $\mathrm{I}$ o I per cent. Since the recombination observed in $T++t f$ heterozygotes was $8 \cdot 3 \pm \mathrm{I} \cdot 5$ per cent. (Lyon, I 956) $t^{6}$ clearly suppresses crossing-over in the $T-t f$ chromosome region.

\section{(ii) Crossing-over in heterozygotes for tf and $\mathrm{t}^{13}$}

Three $T t^{13}++^{t f}$ males, kindly given by Professor L. C. Dunn, were crossed with $T+t f t f$ females and $T t^{13}+t f$ progeny were backcrossed to $++{ }^{T}$ tftf animals. The results are shown in table I. The observed recombination is I I $0 \pm 2 \cdot 00$ per cent. in females and $2 \cdot 9^{2} \pm$ I 44 per cent. in males. A test for heterogeneity of the proportions of recombinants and non-recombinants in the two sexes gives $x^{2}=7 \cdot 62$, $\mathrm{P}<\mathrm{o} \cdot \mathrm{or}$. No such sex difference in recombination was observed in $T++t f$ heterozygotes. For comparison, the data given in the earlier 
paper on tufted, together with. some additional data are included in table I. In this case male heterozygotes show slightly higher recombination, but the difference does not approach statistical significance. Hence, $t^{13}$ clearly has some effect on crossing-over in the $T-t f$ region, but it cannot be considered as a crossover suppressor.

TABLE I

Recombination in the $\mathrm{T}-\mathrm{tf}$ region in $\mathrm{t}^{6}$ and $\mathrm{t}^{13}$ heterozygotes

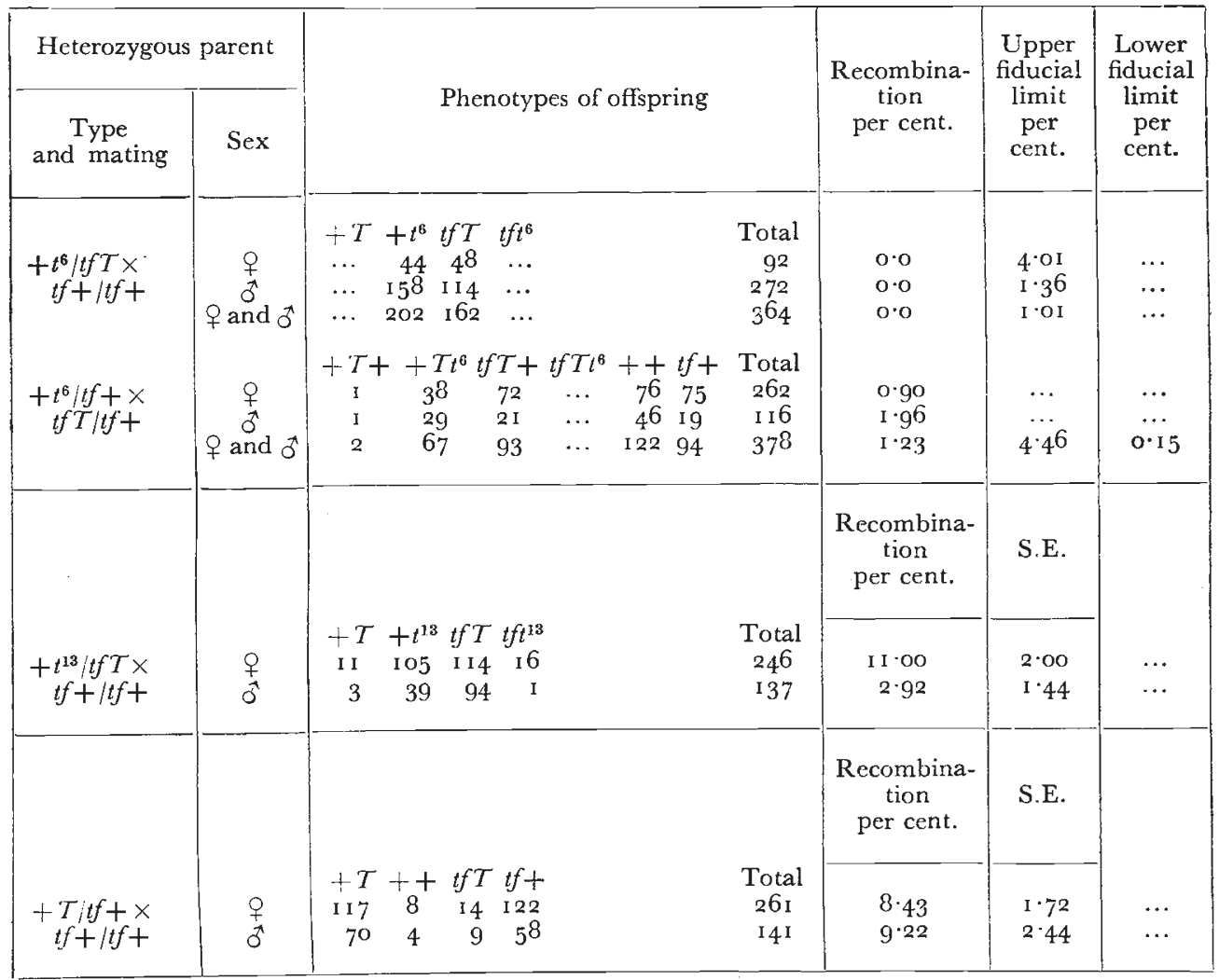

(iii) The effect of $\mathrm{t}^{6}$ on crossing-over in TI38 heterozygotes

In translocation $\mathrm{T}_{13} 8$ the chromosome break in linkage group II is about 20 centimorgans from dilute, $d$, and in group IX the position of the break is such that $\mathcal{T}$ and $d$ show 40 per cent. recombination in translocation heterozygotes (Carter, Lyon and Phillips, I955, I956). Tests were made to find whether $t^{6}$ suppresses crossing-over between $\mathcal{T}$ and the translocation break, by linkage tests between $T$ and $d$ in the presence and absence of $t^{6}$. The tests were designed to run concurrently, and to yield similar phenotypic classes, to minimise viability disturbances in the linkage data. The types of heterozygotes used and results obtained are shown in table 2.

In female heterozygotes carrying $t^{6}$ there is a clear reduction in crossing-over between $T$ and $d$, from $42 \cdot 5 \pm 7 \cdot 8$ per cent. in the absence 
of $t^{6}$ to $22 \cdot 2 \pm 3 \cdot 4$ per cent. in its presence. In male heterozygotes there is again a reduction, but a smaller one, from $40 \cdot 7 \pm 3.9$ per cent. to $32 \cdot 4 \pm 3 \cdot 9$ per cent. To find whether the suppression of crossingover between the $T$ locus and the translocation break was complete, female progeny of these crosses were mated to normal males and tested for semi-sterility by counts of live and dead embryos to detect the presence of the translocation. (The criteria used for diagnosing semisterility were those of Carter, Lyon and Phillips, I955.) Knowing the genotype of the tested animals with respect to $T$ and $d$, the $T-T_{13} 8$

TABLE 2

Recombination in $\mathrm{T}_{\mathbf{3}} \mathbf{8}$ heterozygotes in the presence or absence of ${ }^{\mathrm{t}}$. All matings were backcrosses to $\mathrm{T}_{\mathbf{1}} \mathbf{8} \mathrm{T}_{\mathbf{1}} \mathbf{8}++\mathrm{T}_{\mathrm{dd}}$ animals

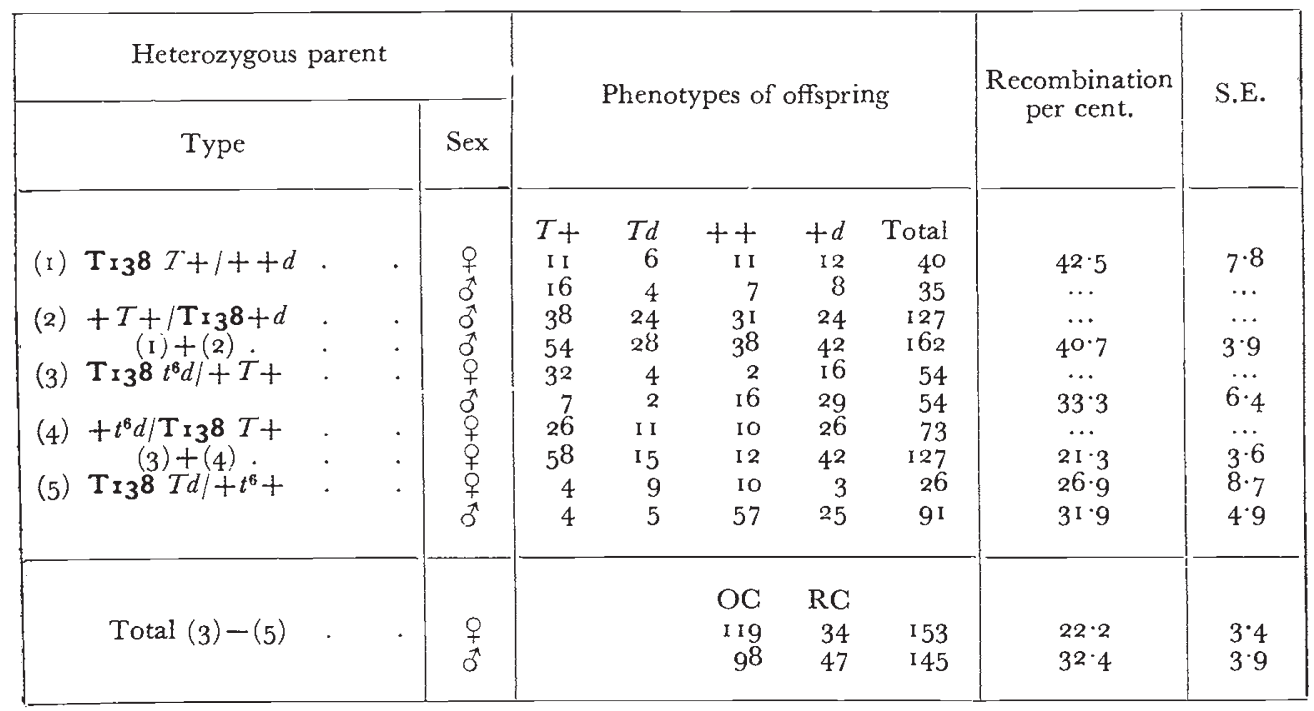

$\mathrm{OC}=$ old combinations $; \mathrm{RG}=$ recombinations.

and $d-\operatorname{Tr}_{3} 8$ recombinations could then be found. This method of estimating linkage by finding the fertility of female progeny gave only an approximate estimate of the recombination since there are two sources of error in testing females for semi-sterility. First, Carter, Lyon and Phillips estimated that I per cent. of wrong diagnoses would be made if only one uterus were examined using their criteria, and second, an appreciable proportion of females cannot be diagnosed either as semi-sterile or as fully fertile since they have an intermediate proportion of dead embryos and come under the category of "test inconclusive". It is likely that these inconclusive animals will not include equal proportions of actual semi-steriles and fully fertiles and this may lead to disturbance of the observed ratios. Table 3 shows that in the presence of $t^{6}$, crossing-over between $T$ and $T_{1} 3^{8}$ still occurs, indicating that the crossover-suppressing effect of $t^{6}$ does not extend throughout the chromosome. The $d-\mathrm{T}_{\mathbf{1}} 38$ recombination shows no significant difference in $T+$ and $T t^{6}$ heterozygotes ; so there is no evidence that 


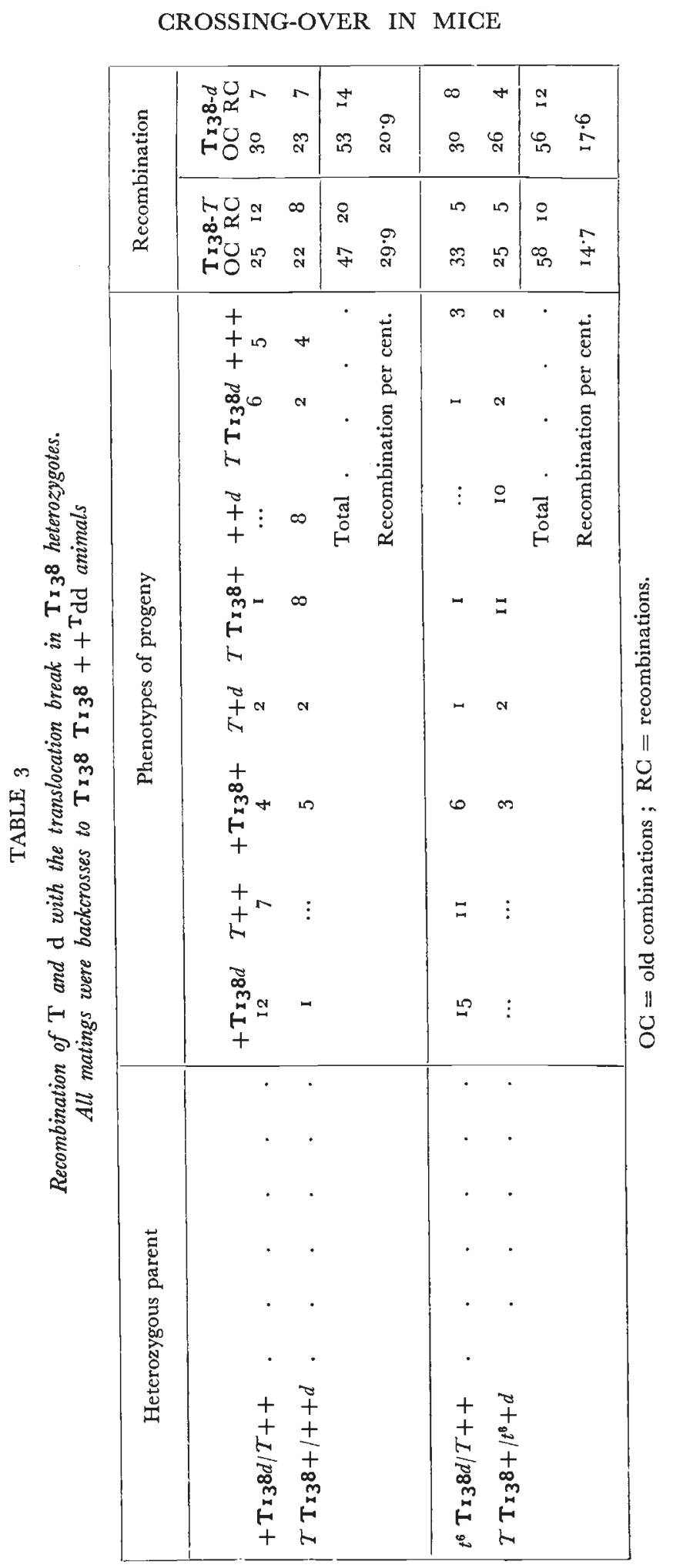


the presence of $t^{6}$ alters crossing-over in arms of the translocation other than that in which $T$ lies.

\section{(iv) The position of $\mathrm{T}$ and $\mathrm{tf}$ relative to the translocation break} in T138

A $T+t f t f$ female was crossed with a Tr38 Tr38dd male, and $T+$ progeny were backcrossed to $++{ }^{T} t f t f d d$ animals. Since $T$ shows approximately 30 per cent. crossing-over with the Tr38 break and $t f$ shows only $8 \cdot 3$ per cent. recombination with $T$, the order of loci could be expected to be either $T-t f-\mathrm{Tr}_{3} 8$ break $-d$ or $t f-T-\mathrm{Tr}_{3} 8$ break $-d$. The two possibilities could be differentiated by the fact that the least frequent classes of offspring could be taken to represent double crossover types. In this case the least frequent classes are $+t f d$ and $T++$ (table 4$)$. These will be double crossovers if the order of loci is $T-t f-\mathrm{Tr}_{\mathbf{3} 8}$ break $-d$ but only single crossovers if the order is $t f-T-\mathrm{T}_{\mathbf{3} 3} 8$ break $-d$. The order is therefore shown to be $T-t f-$ Tr38 break $-d$. This was confirmed by tests of female progeny for Tr38; there were no animals in the classes $+t f \operatorname{Tr}_{38}$ and $T++$, which represent double crossovers if the order is $T-t f-\mathrm{Tr}_{3} 8$ break $-d$.

One unexpected feature of the results is that the $T-t f$ recombination is enhanced in both sexes by the presence of the translocation. The observed recombination was $16.7 \pm 2.9$ per cent. in comparison with the $8 \cdot 3 \pm \mathrm{I} \cdot 5$ per cent. previously observed in $T++t f$ heterozygotes.

\section{(v) Crossing-over between $\mathrm{t}^{6}$ and the translocation break in translocation T190}

Carter, Lyon and Phillips (r955) showed that translocation Trgo is closely linked to $t^{6}$ and reported one doubtful crossover between Trgo and $t^{6}$ in 102 tested animals. Further breeding has resulted in the discovery of two definite crossovers, one of the type Trgo $T /++$, which arose from a cross of a $T \mathbf{r g o} t^{6} t+T$ male by a wild-type female, and one of the type $+^{\text {Troo }} t /++$, which arose from a cross of a Trgo $t^{6} /+T$ female by a wild-type male. This latter crossover was of great interest since it proved to be a $t$ - mutant as well as a crossover. A descendant of the original crossover animal, believed to be of the genotype $+t^{6}$, was given to Professor Grüneberg. Crosses made by Professor Grüneberg of this animal to $T t^{6}$ showed it to be in fact homozygous for a viable $t$-allele, for which the symbol $t^{21}$ is proposed. The evidence for the genotype of this animal is as follows :

\begin{tabular}{|c|c|c|c|}
\hline \multirow{2}{*}{ Parent mating } & \multicolumn{3}{|c|}{ Progeny } \\
\hline & Short tailed & Tailless & Normal \\
\hline$t^{21} t^{21} q \times T t^{6} \sigma^{\uparrow}$ & $\ldots$ & 12 & 20 \\
\hline$t^{6} t^{21} q \times T t^{21} \sigma^{-1}$. & I ? & 80 & 73 \\
\hline
\end{tabular}




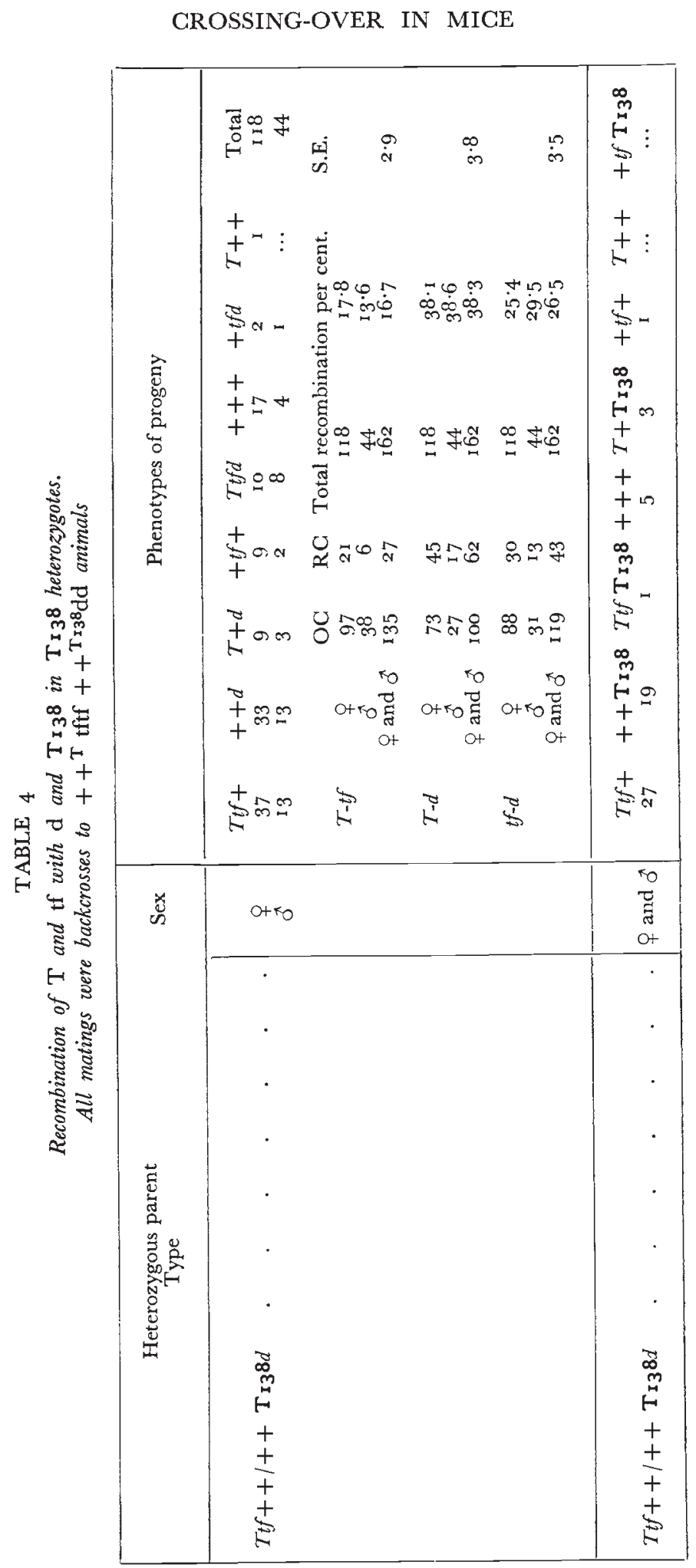


If the female in the first cross had been $+t^{6}$ as expected, then the second crosses would have been $+t^{6} \times T t^{6}$ and in both types of cross shorttailed offspring should have been found,

In linkage tests with Trgo $T /++$ heterozygotes derived from the first crossover animal the number of recombinants was two out of fifteen animals tested, indicating that $t^{6}$ had, as supposed, been suppressing crossing-over between the $\mathrm{T}$ rgo break and $T$.

\section{DISCUSSION}

It has been shown that $t^{6}$ suppresses crossing-over in the $T-t f$ region. But $t^{6}$ had previously been shown not to differ from $t^{0}$, and $t^{0}$ had been shown to suppress crossing-over between $T$ and $F u^{k i}$. Dunn (1958) has recently shown, what was not known at the start of this experiment, that $t f$ lies on the same side of $T$ as $F u^{k i}$ and very close to $F u^{k i}$. Hence it is still unknown whether the crossover-suppressing effect of $t^{6}$ extends on both sides of $T$. The effect of Tr38 heterozygotes, where the presence of $t^{6}$ reduces $T-d$ recombination from 42 per cent. to 22 per cent. in females, suggests at first that the crossoversuppressing action extends further than had previously been known. However, the observation of enhanced crossing-over between $T$ and $t f$ in $\mathrm{T}_{13} 8$ heterozygotes suggests that the region of crossover suppression is in fact quite short in the normal animal but that the translocation, by altering the chiasma localisation in the region, has caused an increased frequency of crossing-over in TI38 heterozygotes not carrying $t^{6}$.

An interesting and unexpected observation was the occurrence of rare crossovers in $+t^{6}+t f$ heterozygotes and in $T_{\text {I9o }}$ heterozygotes. In the $T-t f$ region the observed recombination was $0 / 364$ in $T t^{6}$ $+t f$ heterozygotes and $2 / 162$ in $+t^{6}+t f$ heterozygotes, giving a combined total of $2 / 526$. The frequency of mutants in balanced lethal lines carrying $t$-alleles is $\mathrm{I} / 500$ (Dunn, I956). Crossing-over in the $T-t f$ region in $t$-heterozygotes is thus of the same order of frequency as that of the mutation of these alleles. Furthermore, three of the four crossovers found in $t$-heterozygotes were of the type carrying the $T$ or + allele; the only one of the complementary type, that in Trgo which carried the $t$-allele, was found to carry a mutant $t$. Two explanations for such an occurrence are possible. First, that the presence of a $t$-allele induced a mutational change in the homologous chromosome, carrying $t f$, and that no true crossing-over occurred; and second, that the phenotypic and genetic effects of $t$-alleles are due to an abnormal chromosome segment in which rare crossing-over occurs and, by changing the length or position of the abnormal region, causes a change in the properties of the $t$-allele which is observed as a mutation. The fact that the mutation rate in balanced lethal $t$-stocks is so much higher than the spontaneous mutation rate at other mouse loci, and at the same time comparable with the recombination frequency in this chromosome region lends support to the second hypothesis. Such a hypothesis has been put forward previously by Grüneberg (I952). 
Although the present data lend support to the hypothesis that mutation of $t$-alleles is in fact a result of crossing-over in an abnormal chromosome region, they are clearly insufficient to establish this point. It is important that more animals of the critical crossover type should be found ; it is hoped that an experiment now in progress may yield some of these animals as a by-product. The experiment is intended to find the effect of radiation on mutation in a $T t^{6}$ balanced lethal stock. The stock carries $t f$ heterozygously and hence tests of the $t f$ genotype of mutant animals will show whether or not crossingover has occurred simultaneously.

The hypothesis leads to the expectation that mutant $t$-alleles derived from the original $t^{0}$ and $t^{1}$ stocks still carry some part of the original chromosomal aberration and this may be expected to have some effect on crossing-over in their region, even if they are not complete crossoversuppressors. It is interesting in this connection that $t^{13}$, a mutant derived from $t^{1}$, appears to cause a sex-difference in crossing-over not usually found in this region. A further expectation is that the rare $T$ crossovers found among the progeny of $T t^{6}+t f$ heterozygotes should carry some part of the abnormal chromosome region and should show some of the properties of $t$-alleles. Unfortunately, the crossovers of this type found so far have not been kept.

The original aim of these experiments was to find a crossoversuppressor suitable for use in mutagenetic studies. This aim has not been realised since the chromosomal region in which crossing-over is known to be suppressed by $t^{6}$ still remains short. Nevertheless, the new information gained about crossing-over in $t^{6}$ and $t^{13}$ heterozygotes is such as to open up a new approach to the problem of crossing-over in mice carrying $t$-alleles.

\section{SUMMARY}

The experiments were aimed at extending knowledge of the crossover-suppressing effect of $t$-alleles in the hope that they might prove useful in making special stocks for studies in mutagenesis.

The lethal allele $t^{6}$ suppresses, almost but not quite completely, crossing-over between brachyury, $T$, and the recently found locus of tufted, $t f$.

The allele $t^{13}$, which is not lethal when homozygous, does not suppress crossing-over in this region, but in $T t^{13}+t f$ heterozygotes there is a sex-difference in crossing-over which is not seen in $T++t f$ heterozygotes.

Tufted was found to lie between $T$ and the $T_{13} 8$ translocation break, and the $T-t f$ recombination in $\mathrm{T}_{138}$ heterozygotes was enhanced. $t^{6}$ reduces crossing-over between $T$ and the translocation break in translocation $T_{138}$, and the amount of reduction is such as to make it appear that the crossover-suppressing effect extends little, if at all, beyond $t f$. The effect of $t^{6}$ on crossing-over on the side of $T$ remote from $t f$ is still unknown. 
The frequency of the rare crossovers in the progeny of $t^{6}-t f$ heterozygotes is of the same order as that of the mutant $t$-alleles found in balanced lethal $t$-stocks. Moreover, one of the rare crossovers found between $t^{6}$ and the translocation break in Tigo was discovered to be a mutant as well as an apparent crossover. In view of these two facts the possibility is discussed that the high rate of mutation in balanced lethal $t$-stocks may in fact be due to crossing-over in an abnormal chromosome region.

Acknowledgments.- The authors are grateful to Professor L. C. Dunn for his interest and for the gift of mice carrying $t^{13}$, and to Miss M. L. Court for technical assistance.

\section{REFERENCES}

CARTer, T. C., LYON, M. F., AND PHILlips, R. J. s. I955. Gene-tagged chromosome translocations in eleven stocks of mice. F. Genet., 53, I 54-1 66.

CARTER, T. C., LYON, M. F., AND PHILliPs, R. J. S. 1956. Further genetic studies of eleven translocations in the mouse. 7 . Genet., 54, 462-473.

CARTER, T. C., AND PHILliPS, R. J. S. I950. Three recurrences of mutants in the house mouse. 7. Hered., 4I, 252.

DunN, L. C. 1956. Analysis of a complex gene in the house mouse. Cold Spring Harbor Symp., 21, 187-195.

DUnN, L. C. 1958. Private communication : Mouse Neres Letter No. 18.

DUNN, L. C., AND CASPARI, E. I945. A case of neighbouring loci with similar effects. Genetics, 30, 543-568.

DUNN, L. C., AND GLUECKSOHN-SCHOENHEIMER, s. 1950. Repeated mutations in one area of a mouse chromosome. Proc. Nat. Acad. Sci., 36, 233-237.

DUNN, L. C., AND GLUECKSOHN-WAELSGH, s. I953a. Genetic analysis of seven newly discovered mutant alleles at locus $T$ in the house mouse. Genetics, 38,26 I-27I. DUNN, L. G., AND GLUEGKSOHN-WAELsGH, s. 1953 $b$. The failure of a $t$-allele $\left(t^{3}\right)$ to suppress crossing-over in the mouse. Genetics, $3^{8}, 5^{12-517}$.

GRÜNEBERG, H. I952. The Genetics of the Mouse, 2nd edition. The Hague : Martinus Nijhoff.

LYON, M. F. 1956. Hereditary hair loss in the tufted mutant of the house mouse. F. Hered., 47, гог-103. 